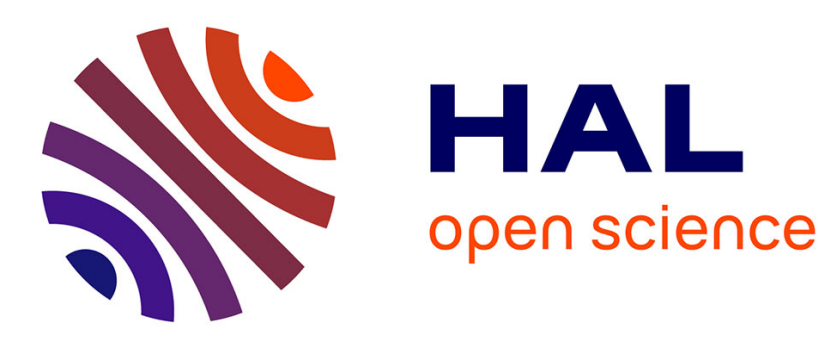

\title{
L'Infomobile à Paris 8: pour une meilleure appropriation de la BU par les usagers
}

Lola Mirabail, Stéphane Tonon

\section{To cite this version:}

Lola Mirabail, Stéphane Tonon. L'Infomobile à Paris 8: pour une meilleure appropriation de la BU par les usagers. Héloïse Courty. Développer l'accueil en bibliothèque: un projet d'équipe, Presses de l'ENSSIB, 2017, Boîte à outils, 978-2-37546-056-6. hal-03518261

\section{HAL Id: hal-03518261 \\ https://hal-normandie-univ.archives-ouvertes.fr/hal-03518261}

Submitted on 9 Jan 2022

HAL is a multi-disciplinary open access archive for the deposit and dissemination of scientific research documents, whether they are published or not. The documents may come from teaching and research institutions in France or abroad, or from public or private research centers.
L'archive ouverte pluridisciplinaire HAL, est destinée au dépôt et à la diffusion de documents scientifiques de niveau recherche, publiés ou non, émanant des établissements d'enseignement et de recherche français ou étrangers, des laboratoires publics ou privés. 


\section{L'Infomobile à Paris 8 : pour une meilleure appropriation de la BU par les usagers}

par Lola Mirabail et Stéphane Tonon

\section{Une politique d'accueil des publics diversifiée}

Soucieux de développer des services adaptés pour répondre aux besoins de ses usagers, le service commun de la documentation (SCD) de l'université Paris 8 Vincennes - Saint-Denis a été récompensé pour la qualité de son accueil par l'obtention du label Marianne en 2014 et la confirmation de son maintien en décembre $2015^{1}$. Regroupé sur un seul site, le SCD de l'université Paris 8 Vincennes - Saint-Denis accueille en moyenne environ 2000 visiteurs par jour. Il offre à son public un espace de $15000 \mathrm{~m}^{2}$, dont environ 11000 dédiés aux espaces publics. Tout en assumant pleinement son rôle de $\mathrm{BU}$, la bibliothèque de Paris 8 joue également un rôle plus vaste d'établissement culturel situé en banlieue et ouvert à toute personne majeure ou titulaire du baccalauréat. La BU pratique, en effet, une politique volontariste en termes d'ouverture au public et propose un système de prêt gratuit à toute personne résidant, étudiant ou travaillant en Île-de-France (1 549 inscrits en 2015 à ce titre). Pour aider cette grande diversité d'usagers à se repérer dans cet espace très vaste, la bibliothèque développe une politique d'accueil des publics dynamique et diversifiée.

Un des services mis en place par la BU depuis 2001 pour répondre aux besoins de son public est l'Infomobile. Ce service vise à aller au-devant des usagers de la bibliothèque, afin de leur expliquer le fonctionnement de la bibliothèque et de les accompagner dans leurs démarches documentaires. Le principe est simple : durant la journée, des personnels de la bibliothèque se déplacent dans les espaces sur des plages de service public d'une heure afin de répondre autrement aux besoins des lecteurs. Ce service est conçu comme un service complémentaire des autres postes de service public, de la formation des usagers et des visites. L'originalité de l'Infomobile tient à la manière dont le dialogue s'engage avec l'usager. Faire du service mobile dans la bibliothèque, c'est aller au-devant des attentes, besoins, incompréhensions, formulés ou non, des usagers. C'est aussi prévenir des usages qui vont à l'encontre du règlement des services.

\section{Les finalités de l'Infomobile}

Lors de l'ouverture de la bibliothèque en 1998, la mise en place de ce « service de très grande proximité ${ }^{2}$ est apparue rapidement essentielle. La mise à disposition directe de la documentation dans les salles et la libre utilisation des postes informatiques invitent le lecteur

${ }^{1}$ Lola Mirabail, «Label Marianne », Bulletin des bibliothèques de France, 2016, nº 8, pp. 124129. [En ligne] < http://bbf.enssib.fr/matieres-a-penser/label-marianne_66389 >.

${ }^{2}$ Michel Dargaud, «Un service de très grande proximité », Bulletin des bibliothèques de France, 2003, n ${ }^{\circ}$ 4, pp. 72-76. [En ligne] < http://bbf.enssib.fr/consulter/bbf-2003-04-0072-014 >. 
à une autonomisation croissante. Ce nouvel espace Bibliothèque entraîne une distanciation spatiale entre l'usager et le bibliothécaire. Certains usagers n'osent pas venir jusqu'aux banques d'information pour poser des questions aux bibliothécaires, d'autres pensent pouvoir se repérer par eux-mêmes tandis qu'ils ne disposent pas de toutes les clefs pour y arriver. Pour réduire cette distance et mieux aider les usagers, il est nécessaire de ne plus adopter une démarche attentiste - attendre que le lecteur vienne poser sa question - mais au contraire de se déplacer, d'adopter une démarche proactive. Cette autonomisation des usagers et la distanciation lecteur-bibliothécaire s'accroissent avec l'installation d'automates de prêt/retour et le passage à la RFID à la rentrée 2014.

La circulation des agents de la bibliothèque permet ainsi de réduire la distance avec les lecteurs et de contourner leurs craintes («je n'osais pas venir vous déranger»). Cette distance est plus importante pour les lecteurs ayant un sentiment d'illégitimité, une impression d'étrangeté face à l'institution bibliothèque ; celle-ci pouvant les impressionner s'ils n'avaient pas l'habitude de s'y rendre auparavant. Ces usagers oseront encore moins aller vers les bibliothécaires de peur de les importuner ou de ne pas être à leurs places. Le service Infomobile a donc tout lieu d'être dans un territoire comme la Seine-Saint-Denis ${ }^{3}$.

Ce service permet d'accompagner les lecteurs dans leur processus d'autonomisation et de prise en main de la BU. Il est complémentaire à la formation des usagers. Il ne s'agit pas d'une formation théorique à tel ou tel outil ou service, pas plus que d'une réponse stricte à la question posée, mais plutôt d'une formation en situation où l'usager, parce qu'il est en demande, sera plus réceptif aux informations qu'on peut lui offrir. Il s'agit d'aller le plus loin possible dans le cadre de la question posée, et, en fonction du lecteur et de la situation, de nouer solidement le lien entre une situation concrète et une compétence plus générale.

L'Infomobile permet aussi d'affirmer la présence des bibliothécaires dans les espaces et de mieux identifier le bibliothécaire comme un médiateur. Lors de leurs interactions avec les lecteurs, les "Infomobiliens" veillent à rappeler aux usagers que, s'ils le souhaitent, des bibliothécaires sont présents pour répondre à leurs questions. Ce service profite ainsi au service public plus traditionnel (derrière des banques). L'Infomobile incarne la relation d'accueil qui lie les usagers à leur bibliothèque.

Enfin l'Infomobile permet également de rappeler les règles à respecter dans la bibliothèque (ne pas manger, ne pas téléphoner, etc.). Cependant, il s'agit seulement de faire un rappel à la règle : «l'Infomobilien » ne se substitue pas au responsable de service public ou aux agents de sécurité.

${ }^{3}$ La bibliothèque accueille en effet une grande diversité de lecteurs. Certains d'entre eux sont issus de milieux très populaires et ne maitrisent pas forcément les codes d'usage d'une bibliothèque. 


\section{Un service institutionnalisé : I'organisation de l'Infomobile}

L'Infomobile est pleinement intégrée dans l'organisation du service public. Elle est rattachée institutionnellement au département des services au public. Clarisse Grasset, la responsable adjointe du service de l'accueil, un des trois services du département, en est la coordinatrice. Les plages d'Infomobile sont considérées comme des plages de service public, et, à ce titre, comptabilisées comme telles.

L'équipe chargée d'assurer l'Infomobile est composée de 3 emplois étudiants, qui assurent chacun 10 heures de service hebdomadaire de la fin septembre à la fin du printemps, ainsi que d'agents de la bibliothèque toutes catégories confondues (A, B et C). En 2015-2016, 5 agents de la bibliothèque effectuaient chacun deux heures de service mobile hebdomadaire. Auparavant, 4 agents de la BU participaient à ce service.

Avant chaque rentrée, les agents font des vœux d'affectation à des postes de service public (accueil, salle thématique, périodiques, audiovisuel, permanence informatique, Infomobile, etc.). Tous les agents de la BU qui le souhaitent ${ }^{4}$, toutes catégories confondues, peuvent émettre le souhait d'être posté, à raison de deux heures par semaine divisées en deux plages, en Infomobile. Ce service connaît beaucoup de succès au sein de l'équipe de la bibliothèque, il est pleinement intégré dans la culture professionnelle de l'établissement. Même si tous les agents ne désirent pas y participer, il y a plus de demandes de participation que de plages disponibles. Pour satisfaire tout le monde, un système de roulement est instauré pour qu'un agent ne fasse pas de l'Infomobile durant deux années consécutives (seule la coordinatrice de l'Infomobile occupe tous les ans ce poste de service public).

Les plages d'Infomobile sont inscrites dans le planning général de service public. Comme pour les autres postes de service public, les agents faisant de l'Infomobile respectent les horaires qui leur sont attribués et qui sont les mêmes toute l'année. Les plages d'Infomobile sont concentrées aux heures de plus fortes affluences du public : durant l'année universitaire, elles avaient lieu de 11 heures à 17 heures, tous les jours de la semaine, sauf le week-end.

Trois vacataires étudiants sont recrutés chaque année pour effectuer des plages d'Infomobile. Leur « double-casquette ${ }^{5}$ (étudiant et «Infomobilien ») est bénéfique au service car ils n'ont pas les mêmes types de relations avec les usagers qui peuvent plus facilement s'identifier à eux et qui osent parfois plus aller vers eux. Ce n'est en revanche pas toujours le cas : certains étudiants préfèrent avouer leurs lacunes à des professionnels plutôt qu'à des pairs. Une composition mixte de l'équipe d' "Infomobiliens" permet ainsi de satisfaire tous les usagers. La coordinatrice se charge de former les vacataires étudiants pour qu'ils maîtrisent l'environnement Bibliothèque: fonctionnement général, outils documentaires, services, espaces, Classification décimale universelle (CDU).

\section{Les compétences à maîtriser pour faire de l'Infomobile}

La coordinatrice de l'Infomobile, Clarisse Grasset, veille à ce que tous les agents postés en Infomobile aient les mêmes connaissances, les mêmes consignes et les mêmes compétences.

${ }^{4}$ Sauf le personnel administratif et les informaticiens qui ne font pas de service public.

${ }^{5}$ L'Infomobile : un service du SCD de Paris 8 Saint-Denis, Thiphaine Tugault, juin 2009.

Commentaire [CS1]: La référence en note de bas de page correspond-elle à un travail d'étudiant? Si oui, est-il possible d'avoir plus de précisions : type de mémoire, nom du directeur ou de la directrice du mémoire, Université... 
Des réunions de rentrée sont organisées et deux guides sont mis à disposition des «infomibiliens» sur l'intranet de la bibliothèque: un guide de formation des nouveaux « infomibiliens », détaillant les compétences nécessaires pour faire de l'Infomobile, et un guide pratique.

Outre une excellente maitrise de la bibliothèque (ses espaces, ses outils, son environnement), il est nécessaire de disposer de compétences particulières d'ordre relationnel. Pour qu'elles puissent être acquises facilement, elles ont été formulées et explicitées : si des qualités peuvent être innées pour certains, il est parfois nécessaire de proposer des astuces à d'autres pour qu'ils puissent s'améliorer dans leurs relations avec les usagers : par exemple, prendre son temps avant de quitter les lieux: un lecteur qui a refusé votre aide l'instant d'avant peut rapidement changer d'avis, ou encore avoir une phrase d'attaque toute prête: "Bonjour. Vous trouvez ce que vous voulez?", "Bonjour vous semblez ne pas trouver ce que vous cherchez. Puis-je vous aider? »...

\section{Repérer des usagers en difficulté}

Les agents doivent tout d'abord savoir repérer les usagers en difficulté afin de pouvoir leur apporter une aide. Pour ce faire, il est conseillé aux agents de :

- faire une ronde systématique dans l'ensemble des salles;

- prendre le temps d'observer ce que font les usagers sans pour autant les espionner ;

- analyser les situations. Le guide de formation distribué aux agents faisant de l'Infomobile donne ainsi plusieurs indices pour repérer un lecteur perdu : « un lecteur perdu» :

o a le nez en l'air dans les rayons;

o a le doigt qui court le long des cotes CDU des ouvrages;

o semble chercher des yeux une signalétique ;

o fixe pendant de longues minutes son écran sans toucher ni souris ni clavier;

o multiplie sans succès les requêtes sur un catalogue ou une base de données ;

o essaie d'emprunter plusieurs fois un livre sur un automate.

\section{Savoir aborder un usager}

Pour être un bon « infomobilien ", l'agent doit savoir aborder un usager sans qu'il se «ferme " devant cette démarche inhabituelle et intrusive. Pour ce faire, il doit :

- porter un badge «Infomobile» ou «Information» bien en évidence; être souriant, avenant et poli ;

- avoir une phrase d'introduction toute prête. Par exemple : «Bonjour. Vous trouvez ce que vous voulez?», «Bonjour vous semblez ne pas trouver ce que vous cherchez. Puis-je vous aider?»; 
- en cas de refus d'aide du lecteur, reformuler sa proposition d'aide en analysant les raisons de son refus : «Ne vous inquiétez pas, je travaille à la bibliothèque et je suis là pour vous aider en cas de besoin" (surprise, gêne, peur d'être abordé, sentiment d'être dragué, n'a pas vu le badge, etc.) ; «Il semble que vous n'arriviez pas à faire $\mathrm{X}$ ou Y sur l'outil A ou B...» « Je travaille à la bibliothèque et je peux vous aider si vous voulez» (sentiment d'être autonome, autre, etc.); ou plus simplement : "vous êtes sûr?»;

- prendre son temps avant de quitter les lieux : un lecteur qui a refusé votre aide l'instant d'avant peut rapidement changer d'avis ;

- ne pas quitter le lecteur sans une petite phrase qui insiste sur la présence de bibliothécaires dans les espaces qui sont au service des usagers : « Si vous avez besoin d'aide, n'hésitez pas à vous adresser aux bibliothécaires qui sont aux banques d'information ou présents dans les salles, ils sont là pour ça, etc. ».

\section{Savoir reformuler les questions posées}

En outre, l'«infomobilien» doit être capable de formuler ou de reformuler la question ou le problème qui se cache derrière l'apparente difficulté repérée. Rares sont les situations où l'Infomobile intervient à la demande d'un usager. La formulation puis la reformulation du problème auquel est confronté le lecteur partent de notre simple impression qu'il est en difficulté, sans qu'il l'ait encore explicitement admis. La (re)formulation est donc plus nécessaire encore qu'en service posté. La bonne compréhension du problème auquel est confronté l'usager est d'autant plus indispensable que c'est l'agent qui la sollicite: ne pas répondre à une question ou répondre à côté risquerait de parasiter l'image du bibliothécaire comme médiateur à laquelle l'Infomobile était en train de donner corps.

Il est enfin important d'avoir suffisamment de pédagogie pour permettre à l'usager non seulement d'avoir une réponse à sa question, mais surtout de s'approprier telle ou telle compétence dont il aura besoin dans la bibliothèque afin qu'il puisse être pleinement autonome s'il le souhaite.

Encadré. Le guide pratique de l'Infomoblie : des situations d'interventions

Il recense les situations fréquemment rencontrées et des propositions d'intervention pour pouvoir aider les infomobiliens à bien répondre aux usagers :

1. Interventions volontaires sur les postes

- Utilisation du catalogue de Paris 8 :

o l'usager tape trop d'informations, multipliant ainsi les risques d'erreurs;

o l'usager ne tape pas assez d'informations et se retrouve devant une liste de 3000 documents ;

o l'usager introduit plusieurs mots de la même racine (« Espagne espagnol espagnols»); 
o les étudiants ont souvent des bibliographies comportant des références d'articles qu'ils introduisent dans le catalogue, évidemment sans résultats ;

o certains caractères empêchent la recherche d'aboutir ;

o en recherche détaillée, l'usager renseigne trop de champs (éditeur, collection, année, etc.) ;

o le livre n'est pas disponible.

- Aide à l'utilisation d'autres ressources :

o dans le Sudoc, vérifier que la recherche porte sur les bons critères;

o dans certaines bases de données, vérifier que l'usager connaît le fonctionnement du moteur de recherche ;

o pour les livres électroniques, expliquer les différentes options de lecture proposées (lecture en ligne, téléchargements) et les services associés (impressions, copier/coller, marque-pages, etc.);

o sur Google, lorsqu'un sujet de recherche donne des dizaines de milliers de pages, proposer d'utiliser la recherche avancée et de cibler la requête sur le titre de la page ;

o lorsqu'une personne semble recopier à la main des informations trouvées sur Internet, lui rappeler qu'elle peut effectuer des impressions.

2. Interventions volontaires en salle

- La plupart des interventions en salle se font entre les rayonnages. Il s'agit de repérer les personnes qui scrutent les étagères, perplexes, un papier à la main : il est rare qu'elles refusent l'aide proposée ;

- de plus, accompagner une personne dans les rayons pour trouver un livre est aussi l'occasion de lui expliquer le fonctionnement de la CDU ; cela lui permet d'être plus autonome dans la bibliothèque et c'est toujours apprécié ;

- le petit guide pratique donne plusieurs clefs au professionnel pour aider le lecteur lorsqu'un livre n'est pas à sa place en rayon.

3. Être à l'affût de tout ce qui peut poser problème aux lecteurs en salle

- C'est une bonne façon d'éviter la « routine » de l'Infomobile (postes et rayonnages);

- les tables sont-elles suffisamment éclairées ? Le silence est-il respecté ? Y a-t-il quelqu'un au bureau d'information pour répondre aux personnes qui attendent ? Etc.

4. Le rappel au règlement

- Il représente une partie non négligeable des interventions. Du fait de leur importante présence dans les espaces, les agents de l'Infomobile sont régulièrement amenés à réguler les usages. Ils concernent en grande majorité la consommation de nourriture/boisson et l'usage du téléphone portable. 


\section{Évaluation et points forts du service}

Chaque année la bibliothèque récolte des informations ayant trait à l'activité des bibliothécaires en service public au moyen de semaines tests durant lesquelles les agents recensent les questions que leur posent les usagers. Ces semaines tests permettent notamment d'évaluer l'activité de l'Infomobile. En 2015, deux semaines test ont été menées : une en mai, en période de faible affluence, et une en décembre, en période de forte affluence.

Pour la semaine test du mois de décembre, le service représente $7 \%$ de l'activité de la bibliothèque en service public avec 287 lecteurs abordés. En moyenne, 10 lecteurs sont abordés par plage d'une heure par agent. La tranche horaire qui compte le plus d'interventions est la tranche 11 heures- 13 heures $^{6}$. Le taux d'acceptation du service est très fort: environ $89 \%$ des lecteurs acceptent l'aide proposée. Les usagers sont en majorité très satisfaits du service apporté dans le cadre de l'Infomobile.

Les principales activités de l'Infomobile sont l'orientation intellectuelle et documentaire (37\% des questions en décembre et $12 \%$ des questions en mai) et l'orientation géographique/informations générales (31\% en mai et $16 \%$ en décembre). Le service occupe également un fort rôle de contrôle et de régulation ( $21 \%$ en mai et $20 \%$ en décembre).

Cette activité est très stimulante pour les agents et leur permet de mieux connaître les attentes des lecteurs. L'Infomobile est notamment un bon vecteur d'intégration pour les nouveaux arrivants.

Une autre force de l'Infomobile est sa complémentarité avec les autres postes de service public. Les Infomobiliens peuvent être conduits à accompagner des lecteurs vers des banques de renseignement bibliographique afin de mieux les faire connaître. Il permet d'accroître la visibilité des différents postes de service public auprès des usagers (« Si vous avez d'autres questions, sachez que vous pouvez aller à telle ou telle banque »). De même, les infomobiliens peuvent prendre le relais des agents de l'accueil, ce qui permet de désengorger la banque d'accueil en période de forte affluence.

L'Infomobile permet d'appréhender autrement la relation entre le bibliothécaire et l'usager mais aussi celle entre l'agent et l'espace de la bibliothèque. Fort de son succès, ce service est voué à se développer davantage.

Cette nécessité de mobilité concerne également les postes de service public plus traditionnels. Les postures des agents en service public et la manière dont ils occupent l'espace Bibliothèque sont, plus largement, conduites à évoluer dans un contexte d'autonomisation des lecteurs - ou de montée d'un sentiment d'autonomie - croissante. Même si, lors de sa plage de service public (hors Infomobile), l'agent est en grande majorité derrière un bureau (de renseignements, d'accueil...), il est amené de plus en plus fréquemment à se déplacer. À Paris 8, il est ainsi demandé aux agents postés à des banques de renseignements de se déplacer toutes les trente minutes dans leurs salles et les salles voisines pour repérer les lecteurs ayant besoin d'eux, et les problèmes s'il y en a, mais également pour être bien identifiés par les usagers. De même, lorsqu'il est possible de le faire, il est préconisé aux agents d'accompagner

${ }^{6}$ Rapport annuel d'activité 2015, Bibliothèque de Paris 8. [En ligne] < www.bu.univ-paris8.fr >. 
les lecteurs dans les espaces quand la question les y invite (« je ne trouve pas un ouvrage », « le photocopieur ne marche pas », etc.). 